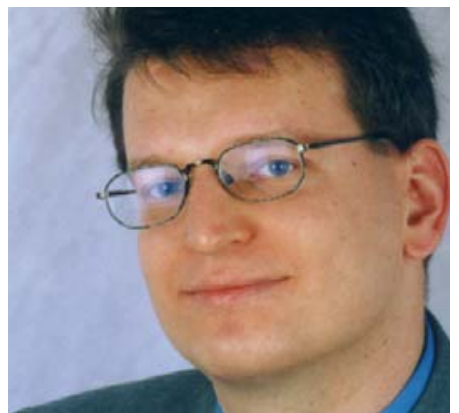

Prof. Dr. med. habil. Jörg Klewer, Zwickau

Schriftleitung heilberufescience@springer.com

HeilberufeSCIENCE 2010; 1 (4): 100 DOI 10.1007/s16024-010-0420-6

\title{
Zwei Jahre HeilberufeSCIENCE
}

Mit der vorliegenden Ausgabe feiert HeilberufeSCIENCE nicht nur den zweiten Geburtstag, sondern komplettiert auch den ersten Jahrgang als eigenständiges Online-Journal. Dieser erste eigenständige Jahrgang umfasst insgesamt 20 Artikel und zwei wissenschaftliche Kurzmitteilungen. Diese werden durch zwei Kongress-Sonderausgaben mit den Beiträgen des Kongresses "Pflege 2010" vom 29.-30.1.2010 in Berlin und des "8. Gesundheitspflege-Kongress" vom 19.-20.11.2010 in Hamburg ergänzt. Somit konnte HeilberufeSCIENCE Ihnen auch im zweiten Jahr regelmäßig ein breites Spektrum an Artikeln und Kongressbeiträgen aus den Gebieten Pflegemanagement, Pflegepädagogik, Pflegewissenschaften, Gerontologie, Altenpflege und Gesundheitswissenschaften/ Public Health bieten. Aufgrund der zunehmenden Anzahl der in der Redaktion eingehenden hochwertigen Beiträge sehe ich der weiteren Zukunft von HeilberufeSCIENCE optimistisch entgegen.

In dieser aktuellen Ausgabe November 2010 wird Ihnen erstmalig ein englischsprachiger Beitrag in Form einer Übersichtsarbeit geboten. Darin präsentieren Kroll und Kehn ein Review zur Wirksamkeit von nicht-pharmakologischen und nichtoperativen Maßnahmen bei der Schmerztherapie von Patienten nach Rückenmarksverletzungen. Sicherlich liegt auch zukünftig der Fokus der meisten Beiträge in HeilberufeSCIENCE im deutschsprachigen Raum, jedoch sollen sich internationale Autoren ebenfalls angesprochen fühlen ihre Beiträge einzureichen.

Des Weiteren gehen in dieser Ausgabe Schaal et al. mittels eines methodisch orientierten Ansatzes darauf ein, wie eine Outsourcing-Analyse für eine Wäscherei einer stationären Altenpflegeeinrichtung durch- geführt werden könnte. Daran schließt sich die Arbeit von Möller et al. an, die sich mit den aktuellen Chancen und Risiken der Qualitätsberichterstattung für die ambulante Pflege auseinandersetzt. Der darauf folgende Beitrag von Elmer et al. betrachtet den Einsatz der Balanced Scorecard in der Pflege und die damit verbundenen Auswirkungen auf die Mitarbeiter. Anschließend präsentieren Hirsch et al. die Ergebnisse einer sehr umfassenden Studie an fast 1.000 Gesundheits- und Krankenpflegeschülern im Bundesland Sachsen-Anhalt zu Rauchverhalten, Alkohol- und Drogenkonsum sowie Impfungen. Den Abschluss bildet die wissenschaftliche Kurzmitteilung von Fleischer et al., die kurz aber prägnant aufzeigt, mit welchen Problemen der Einsatz des "Mini Nutritional Assessment" (MNA) in einer stationären Altenpflegeeinrichtung verbunden sein kann, wenn Pflegefachkräfte nicht ausreichend auf den Gebrauch dieses Assessments vorbereitet werden. Diesmal möchte ich Sie nicht nur einladen, die sechs Beiträge dieser Ausgabe von HeilberufeSCIENCE kritisch zu lesen, sondern auch einen Blick in die fast zeitgleich erscheinende HeilberufeSCIENCE-Sonderausgabe mit den Abstracts der Poster, wissenschaftlichen Vorträge und Fachbeiträge des 8. Gesundheitspflegekongresses zu werfen. Vielleicht finden Sie darin weitere interessante Anregungen für Ihre eigene Tätigkeit.

Ihr
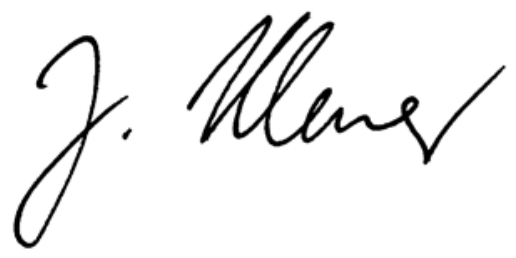